\title{
Delafloxacin: A Review in Acute Bacterial Skin and Skin Structure Infections
}

\author{
L. J. Scott ${ }^{1}$
}

Published online: 14 July 2020

(c) Springer Nature 2020, corrected publication 2020

\begin{abstract}
The global spread of antibacterial-resistant strains, especially methicillin-resistant Staphylococcus aureus (MRSA) for acute bacterial skin and skin structure infections (ABSSSIs), has driven the need for novel antibacterials. Delafloxacin [Quofenix ${ }^{\mathrm{TM}}(\mathrm{EU})$; Baxdela $^{\circledR}$ (USA)], a new fluoroquinolone (FQ), has a unique chemical structure that enhances its antibacterial activity in acidic environments such as occurs in ABSSSIs (including S. aureus infections). Delafloxacin (intravenous and oral formulations) is approved in several countries for the treatment of adults with ABSSSIs (featured indication). In intent-to-treat analyses in pivotal phase 3 trials in adults with ABSSSIs, including those with comorbid disease, intravenous delafloxacin monotherapy ( \pm oral switch after six doses) twice daily was noninferior to intravenous vancomycin + aztreonam for primary endpoints, as specified by the FDA (objective response rate at 48-78 $\mathrm{h}$ after initiation of therapy) and the EMA [investigator-assessed clinical cure rate at the follow-up visit at day 14 ( \pm 1 day)]. Delafloxacin was generally well tolerated, with most treatment-related adverse events mild to moderate in severity and few patients discontinuing treatment because of these events. Relative to vancomycin + aztreonam (a non-FQ regimen), delafloxacin treatment was not associated with an increased risk of FQ-associated AEs of special interest. Given its unique chemical structure that confers novel properties relative to other FQ and its broad spectrum of activity against common clinically relevant Gram-positive pathogens, including against MRSA strains ( \pm FQ-resistance mutations), and Gram-negative pathogens, intravenous delafloxacin ( \pm oral switch) provides a novel emerging option for the treatment of adult patients with ABSSSIs.
\end{abstract}

Enhanced material for this Adis Drug Evaluation can be found at https://doi.org/10.6084/m9.figshare.12218537.

The manuscript was reviewed by: $\boldsymbol{C}$. Adembri, Department of Health Sciences, Section of Anesthesiology and Critical Care, Careggi Hospital, Università di Firenze, Florence, Italy; K. $\boldsymbol{C}$. Carroll, Department of Pathology, Johns Hopkins University School of Medicine, Baltimore, MD, USA; C. El Haj, Department of Infectious Disease, University Hospital Bellvitge, Barcelona, Spain; O. Murillo, Department of Infectious Disease, University Hospital Bellvitge, Barcelona, Spain; R. Zaragoza, Intensive Care Unit, University Hospital Dr Peset, Valencia, Spain.

L. J. Scott

demail@springer.com

1 Springer Nature, Mairangi Bay, Private Bag 65901, Auckland 0754, New Zealand
Delafloxacin: clinical considerations in acute bacterial skin and skin structure infections

Novel anionic FQ, acts via equipotent targeting of the bacterial enzymes topoisomerase IV and DNA gyrase

Potent in vitro activity against a broad spectrum of relevant Gram-positive and Gram-negative bacteria, including those carrying specific resistance mutations such as MRSA ( \pm FQ-resistant mutations)

Noninferior efficacy to vancomycin plus aztreonam and generally well tolerated in pivotal phase 3 trials

Unlike other FQs, not associated with clinically relevant QT prolongation or phototoxicity 


\section{Introduction}

Acute bacterial skin and skin structure infections (ABSSSIs) are heterogeneous, potentially life-threatening infections involving the skin and underlying subcutaneous tissue, fascia or muscle, and include cellulitis/erysipelas, wound infections and major cutaneous abscesses [1]. ABSSSIs are associated with increased morbidity, mortality and duration of stay in hospital, thereby imposing a significant burden from a societal and healthpayer perspective. The most common causative pathogens are Staphylococcus aureus and Streptococcus spp. [1,2]. ABSSSIs may be mono- or polymicrobial (include Gram-positive, Gram-negative and/or obligate anaerobic pathogens) in nature, with polymicrobial infections especially prevalent in patients with comorbidities or those who have failed prior antibacterial treatment. Patients with polymicrobial infections may be at higher risk of inadequate treatment and require broad-spectrum antibacterials [3]. Fluoroquinolones (FQs), given their broad spectrum of activity against clinically relevant Gram-positive and Gram-negative bacteria and availability as intravenous and oral formulations (provides flexibility to transition from intravenous to oral therapy), are amongst the most commonly utilized classes of antibacterials for treating infectious diseases [4]. However, in recent years, the global emergence of methicillin-resistant $S$. aureus (MRSA) strains and other resistant pathogens, including FQ-resistant and multi-drug resistant (MDR) bacteria, has posed a major concern for contemporary healthcare systems [3]. In light of this, there has been a drive to develop novel classes and novel agents within existing classes of antibacterials. Indeed, improved crystallographic techniques and structure-activity studies have played an important role in identifying novel multiple targets within known antibacterial classes (such as FQs) [4-6].

One such chemically unique FQ is delafloxacin [Quofenix ${ }^{\mathrm{TM}}$ (EU); Baxdela ${ }^{\circledR}$ (USA)]. Intravenous and oral formulations of delafloxacin are approved in several countries for the treatment of adults with ABSSSIs (featured indication) $[7,8]$ and/or community-acquired bacterial pneumonia (approved in the USA [7], but not in the EU [8]; discussion of this indication is beyond the scope of this review). This article reviews the clinical use of delafloxacin in adults with ABSSSIs, and summarizes its pharmacological properties and in vitro activity.

\section{Pharmacodynamic Properties of Delafloxacin}

Delafloxacin is a novel fully synthetic anionic FQ, with modifications of the quinolone structure performed to enhance its spectrum of antibacterial activity, pharmacokinetic profile and toxicity profile. Three unique changes in the chemical structure of quinolone differentiate delafloxacin from other FQs: namely, the presence of a substituted heteroaromatic ring at the N1 position (enhances its antibacterial activity vs other FQs), a weak polarity at the $\mathrm{C} 8$ position (postulated to enhance its potency against quinolone-resistant Gram-positive bacteria) and the absence of a basic group at the $\mathrm{C} 7$ position (makes it a weakly acidic molecule, thereby enhancing its antibacterial activity in acidic environments such as in ABSSSIs) [4-6, 9-13].

The antibacterial activity of delafloxacin is due to its dual-targeted equipotent inhibition of the essential bacterial enzymes topoisomerase IV and DNA gyrase (topoisomerase II). Conversely, other FQs (e.g. ciprofloxacin, levofloxacin and trovafloxacin) differ in their relative binding affinity for each these enzymes, exhibiting more potent inhibition of either topoisomerase IV or DNA gyrase. As a consequence of dual targeting, delafloxacin exhibits a broad spectrum of activity against clinically relevant microorganisms and a low risk of the emergence of resistance amongst susceptible pathogens $[4-6,9,10,14]$.

\subsection{Antibacterial Activity}

This section focuses on the in vitro antibacterial activity of delafloxacin against clinical isolates of Gram-positive and Gram-negative bacteria associated with ABSSSIs against which delafloxacin has demonstrated efficacy in clinical trials. In the EU summary of product characteristics [8] and/or US prescribing information [7] specified pathogens are $S$. aureus [including MRSA and methicillin-susceptible (MSSA) isolates] [7, 8], Staphylococcus haemolyticus [7, 8], Staphylococcus hominis [8], Staphylococcus lugdunensis [7, 8], Streptococcus agalactiae [7, 8], Streptococcus anginosus group (including Streptococcus anginosus, Streptococcus intermedius, Streptococcus constellatus) [7, 8], Streptococcus dys galactiae [8], Streptococcus mitis group (including Streptococcus cristatus, Streptococcus gordonii, Streptococcus oralis, Streptococcus mitis, Streptococcus sanguinis) [8], Streptococcus pyogenes [7, 8], Enterococcus faecalis [7, 8], Escherichia coli [7, 8], Enterobacter cloacae [7, 8], Klebsiella oxytoca [8], Klebsiella pneumoniae [7, 8], Proteus mirabilis [8] and Pseudomonas aeruginosa [7, 8]. EUCAST defined minimum inhibitory concentration (MIC) breakpoints for susceptibility of delafloxacin against specified microorganisms are: $S$. aureus $(0.25 \mu \mathrm{g} / \mathrm{mL}) ;$ S. pyogenes, $S$. dysgalactiae, $S$. agalactiae and $S$. anginosus (all $0.03 \mu \mathrm{g} / \mathrm{mL}) ;$ E. coli $(0.125 \mu \mathrm{g} / \mathrm{mL})$ [8]. Emphasis is given to results from recent in vitro studies involving $>500$ clinical isolates collected since 2014 $[15,16]$ (or year not reported $[17,18]$ ). MICs were determined using CLSI broth microdilution methods [15-17]. 
Against clinical isolates collected globally or in the EU and/or USA, delafloxacin exhibited potent in vitro activity (based on MIC required to inhibit the growth of $90 \%$ of isolates; $\mathrm{MIC}_{90}$ ) against a broad spectrum of Gram-positive and Gram-negative pathogens commonly associated with ABSSSIs (Table 1) [15-18]. Delafloxacin also exhibited potent in vitro activity against Gram-positive strains exhibiting resistance to one or more clinically relevant antibacterial drugs, including against MRSA and MSSA strains (both \pm levofloxacin resistance), MR and MS coagulasenegative staphylococci (CoNS), and $S$. pneumoniae strains resistant to ceftriaxone, levofloxacin, penicillin or multiple drugs (Table 1). In the largest of these study, the vast majority of MSSA ( $99.5 \%$ of isolates from both the EU and USA), MRSA (95.3 and 91.2\%), MS CoNS (100 and 97.6\%) and MR CoNS (99.5 and 84.5\%) isolates were inhibited by delafloxacin at an MIC breakpoint of $\leq 0.5 \mu \mathrm{g} / \mathrm{mL}$, with delafloxacin inhibiting $88.3 \%$ (484/548 isolates) of FQ-resistant $S$.

Table 1 In vitro activity of delafloxacin against selected clinical isolates

Pathogen

Total no of isolates $\quad \mathrm{MIC}_{90}(\mu \mathrm{g} / \mathrm{mL}$; range across studies) [\% susceptible; CLSI/EUCAST]

\begin{tabular}{|c|c|c|c|c|}
\hline & & Delafloxacin & Levofloxacin & Vancomycin \\
\hline \multicolumn{5}{|l|}{ Gram-positive isolates } \\
\hline Enterococcus faecalis [15] & 450 & 1 & $>4[70.7 / 70.7]$ & $2[97.8 / 97.8]$ \\
\hline Staphylococcus aureus $[15,17]$ & 2035 & 0.25 & $>4[64.4 / 64.4]$ & $1[100 / 100]$ \\
\hline MRSA $[15,17]$ & 867 & $0.25-0.5$ & $>4[30 / 30]$ & $1[100 / 100]$ \\
\hline MRSA LEV-R [17] & 195 & 0.25 & NR & NR \\
\hline MRSA LEV-S [17] & 101 & 0.008 & NR & NR \\
\hline MSSA $[15,17]$ & 1172 & $0.008-0.03$ & $2[89.8 / 89.8]$ & $1[100 / 100]$ \\
\hline MSSA LEV-R [17] & 39 & 0.25 & NR & NR \\
\hline MSSA LEV-S [17] & 358 & 0.008 & NR & NR \\
\hline S. aureus LEV-R [17] & 232 & 0.25 & NR & NR \\
\hline S. aureus LEV-S [17] & 455 & 0.008 & NR & NR \\
\hline MR CoNS [15] & 125 & 0.5 & $>4[38.4 / 38.4]$ & $2[100 / 100]$ \\
\hline MS CoNS [15] & 75 & 0.06 & $4[88 / 88]$ & $2[100 / 100]$ \\
\hline Streptococcus agalactiae $[15,18]$ & 335 & $0.015-0.03$ & $1[97.8 / 96.9]$ & $0.5[100 / 100]$ \\
\hline Streptococcus anginosus group [18] & 105 & 0.008 & NR & NR \\
\hline Streptococcus dysgalactiae $[15,18]$ & 232 & $0.015-0.03$ & $1[99.2 / 97.0]$ & $0.25[100 / 100]$ \\
\hline Streptococcus mitis group [18] & 101 & 0.06 & NR & NR \\
\hline Streptococcus pneumoniae $[15,16,18]$ & 747 & $0.015-0.03$ & $1[98.9 / 98.9]$ & NA \\
\hline S. pneumoniae CEF-R [16] & 9 & $\leq 0.004-0.015$ & NR & NR \\
\hline S. pneumoniae LEV-R [16] & 30 & 0.5 & NR & NR \\
\hline S. pneumoniae MDR [16] & 82 & 0.015 & NR & NR \\
\hline S. pneumoniae PR [16] & 13 & 0.015 & NR & NR \\
\hline Streptococcus pyogenes $[15,18]$ & 532 & $0.015-0.016$ & $1[99.8 / 96.5]$ & $0.5[100 / 100]$ \\
\hline \multicolumn{5}{|l|}{ Gram-negative isolates } \\
\hline Escherichia coli $[15]$ & 500 & 4 & $>4[69.6 / 69.6]$ & NA \\
\hline E. coli $\mathrm{ESBL}[15]$ & 92 & $>4$ & $>4[21.7 / 21.7]$ & \\
\hline Enterobacter spp [15] & 384 & 1 & $0.5[96.6 / 95.8]$ & NA \\
\hline Klebsiella oxytoca [15] & 111 & 0.12 & $\leq 0.12[100 / 100]$ & NA \\
\hline Klebsiella pneumoniae [15] & 389 & $>4$ & $>4[81.5 / 80.2]$ & NA \\
\hline K. pneumoniae ESBL [15] & 102 & $>4$ & $>4[34.3 / 32.4]$ & NA \\
\hline Proteus mirabilis [15] & 211 & 2 & $>4[78.7 / 71.1]$ & NA \\
\hline Pseudomonas aeruginosa [15] & 200 & $>4$ & $>4[72.5 / 62.5]$ & NA \\
\hline
\end{tabular}

Pathogens against which delafloxacin has demonstrated efficacy in clinical trials [7, 8]. Studies involving $>500$ clinical isolates collected globally in pivotal phase 3 delafloxacin trials [17], globally in the SENTRY program [18], in the EU and USA [15] or in the USA [16] in 2014 [15, 16] (year not reported [17, 18]); not all studies reported all drugs or susceptibilities. Abstract plus poster presentation [18]

$C E F-R$ ceftriaxone resistant, $C L S I$ Clinical and Laboratory Standards Institute, CoNS coagulase-negative staphylococci, $E S B L$ extended-spectrum $\beta$-lactamase, EUCAST European Committee on Antimicrobial Susceptibility Testing, LEV levofloxacin, LEV-R LEV-resistant, $L E V$-S LEVsusceptible, $M D R$ multi-drug resistant, $M I C_{90}$ minimum inhibitory concentration required to inhibit $90 \%$ of isolates, $M R$ methicillin-resistant, MRSA MR S. aureus, MS methicillin-susceptible, MSSA MS S. aureus, NA not applicable, NR not reported, $P R$ penicillin-resistant 
aureus and CoNS isolates at this breakpoint. At a breakpoint of $\leq 0.03 \mu \mathrm{g} / \mathrm{mL}, \approx 99 \%$ of $S$. pneumoniae isolates were inhibited by delafloxacin, with delafloxacin several times more potent than ceftaroline (8-fold), moxifloxacin (16-fold) and levofloxacin (64-fold) [15]. In two global phase 3 trials in adults with ABSSSIs (Sect. 4), similar high microbiological response rates (i.e. eradication or presumed eradication) were observed against levofloxacin-nonsusceptible $S$. aureus (80/91 isolates; 98.8\%) and MRSA isolates (70/71; 98.6\%), as well as against isolates with mutations in the quinolone resistance determining region (QRDR) [17]. Amongst delafloxacin recipients with infections caused by $S$. aureus isolates that had S84L and S90Y mutations in $\operatorname{gyrA}$ and $\operatorname{parC}$, respectively (the most commonly observed QRDR mutations in phase 3 trials), the microbiological response rate was $98.6 \%$ [17].

In vitro, delafloxacin exhibited potent activity at clinically achievable concentrations against biofilms of both MSSA and MRSA, reducing biofilm viability by at least $50 \%$ and also reducing film thickness $[19,20]$. For example, in one of these studies, the biofilm activity of delafloxacin was better than that of daptomycin against MRSA biofilms, with both of these agents more effective than other tested anti-staphylococcal agents, including moxifloxacin, linezolid, rifampin, tigecycline and vancomycin [20]. The more potent activity of delafloxacin, at least in part, reflects its penetration into the acidic microenvironment of the biofilm.

For Gram-negative pathogens, $80.9 \%$ of Enterobacteriaceae strains $\left(n=2250\right.$; delafloxacin $\left.\mathrm{MIC}_{90} 4 \mu \mathrm{g} / \mathrm{mL}\right)$ were inhibited by delafloxacin at an MIC breakpoint of $\leq 1 \mu \mathrm{g}$ / $\mathrm{mL}$, although $90 \%$ of FQ-resistant Enterobacteriaceae strains (i.e. MIC $>1 \mu \mathrm{g} / \mathrm{mL}$ ) exhibited decreased susceptibility to delafloxacin. Delafloxacin demonstrated more potent in vitro activity against non-extended-spectrum $\beta$-lactamase (ESBL)-producing $E$. coli and $K$. pneumoniae than against respective ESBL-producing strains (Table 1). Conversely, delafloxacin demonstrated potent in vitro activity against both nonESBL- and ESBL-producing strains of $K$. oxytoca ( $\mathrm{MIC}_{90} 0.12 \mu \mathrm{g} / \mathrm{mL}$ for both) [15].

In vitro, delafloxacin exhibits concentration-dependent bactericidal activity against Gram-positive and Gram-negative bacteria [7]. Delafloxacin demonstrated bactericidal in vitro activity against strains of MRSA collected in 2013 from patients participating in delafloxacin phase 3 trials in ABSSSI, with minimum bactericidal concentrations of $0.008 \mu \mathrm{g} / \mathrm{mL}, 0.5 \mu \mathrm{g} / \mathrm{mL}$ and $8 \mu \mathrm{g} / \mathrm{mL}$ against MRSA strain 110 (levofloxacin susceptible), 124 (triple mutant) and 165 (quadruple mutant), respectively (vs $0.5,8$ and $>32 \mu \mathrm{g} / \mathrm{mL}$ for levofloxacin) [21]. Against strain 110, bactericidal activity was observed at $6 \mathrm{~h}$ for most concentrations of delafloxacin and levofloxacin; at $16 \times$ MIC, delafloxacin was associated with more rapid killing of MRSA strain 124 than the same concentration of levofloxacin; and at $24 \mathrm{~h}$, delafloxacin was bactericidal at 16 and $32 \times$ MIC against MRSA strain 165 [21].

In vitro drug combination studies showed no synergy or antagonism occurred between delafloxacin and amoxicillin/clavulanate, azithromycin, aztreonam, ceftaroline, ceftazidime, ceftriaxone, colistin, daptomycin, doxycycline, linezolid, meropenem, penicillin, rifampin, tigecycline, trimethoprim/sulfamethoxazole or vancomycin [7].

\subsection{Resistance}

Resistance to FQs, including delafloxacin, can occur due to mutations in defined regions of the target bacterial enzymes topoisomerase IV and DNA gyrase (i.e. QRDRs) or other resistance mechanisms such as altered efflux [7, 8]. Cross-resistance between delafloxacin and other FQs may be observed, although some isolates resistant to other FQs may retain susceptibility to delafloxacin $[7,8]$, including S. aureus isolates carrying mutations in the QRDR ( $g y r A$, gyrC and parE) (Sect. 2.1) [7]. Although delafloxacin may be affected by targeted mutations, elevations in MIC values potentially have a minimal impact, given the intrinsic antibacterial in vitro activity of delafloxacin is markedly higher than other FQs, including moxifloxacin (considered the most potent FQ against Gram-positive pathogens) [22]. $\mathrm{MIC}_{90}$ values for delafloxacin were twofold to fourfold lower than those of moxifloxacin [22]. This difference was more marked at acidic $\mathrm{pH}(\mathrm{pH} 5.5)$, with MIC values for delafloxacin 5-7 dilutions lower than at neutral $\mathrm{pH}$, whereas those for moxifloxacin increased by $2-3$ dilutions at the lower $\mathrm{pH}$ [6]

In vitro, resistance to delafloxacin develops in multiple step mutations in the QDRDs of Gram-positive and Gramnegative bacteria [7]. In vitro, delafloxacin was associated with a low probability of selecting resistance against MRSA strains $\left(2 \times 10^{-9}\right.$ to $\left.<9.5 \times 10^{-11}\right)$ [23].

\subsection{Pharmacodynamic/Pharmacokinetic Considerations}

As for other FQs, the pharmacodynamic/pharmacokinetic relationship most closely associated with the efficacy of delafloxacin was the 24-h free-drug area under the plasma concentration-time curve ( $f$ AUC)/MIC ratio [8]. In a neutropenic murine lung infection model, the median $f \mathrm{AUC} /$ MIC targets associated with net stasis with delafloxacin were 1.42 for $S$. aureus, 0.56 for $S$. pneumoniae and 40.3 for $K$. pneumoniae, with respective 1-log kill targets of 7.92, 3.36 and 55.2 [24]. In a neutropenic murine pneumonia model, against $P$. aeruginosa isolates, the median $f$ AUC/MIC target associated with net stasis with delafloxacin was 5.66, with a 1-log kill value of 14.3 [25]. 


\subsection{Other Effects}

In healthy adult volunteers, therapeutic and supratherapeutic intravenous doses of delafloxacin had no clinically relevant effects on the QT/QTc interval (i.e. no clinically relevant QT prolongation), atrioventricular conduction or cardiac depolarization (as assessed by the PR and QRS interval durations) in two cardiac safety studies (M01-365 and RX-3341$11 ; n=68$ and 52 enrolled) [26, 27].

In healthy adult volunteers $(n=47)$, delafloxacin exhibited no clinically significant phototoxic potential at all wavelengths tested (295-430 nm) after 6 days of oral delafloxacin $200 \mathrm{mg} /$ day or $400 \mathrm{mg} /$ day [28].

\section{Pharmacokinetic Properties of Delafloxacin}

Pharmacokinetic parameters of delafloxacin are similar in patients with ABSSSI to those in healthy volunteers [8]. In healthy adult volunteers, after single and multiple doses of intravenous $(300 \mathrm{mg})$ and oral $(450 \mathrm{mg}$ ) delafloxacin, the pharmacokinetics of the drug were bioequivalent $[8,29]$, supporting the switching regimen (from intravenous to oral formulations) utilized in phase 3 trials [29].

Oral delafloxacin exhibited linear pharmacokinetics across a dose range of 50 to $1600 \mathrm{mg}$ in healthy volunteers [30]. Delafloxacin was rapidly absorbed after intravenous $(300 \mathrm{mg})$ or oral $(450 \mathrm{mg})$ administration, with respective median times to maximum plasma concentration of $0.75 \mathrm{~h}$ and $1 \mathrm{~h}$ after single and multiple (once every $12 \mathrm{~h}$ ) oral doses (vs $1 \mathrm{~h}$ after single and multiple intravenous doses) [7]. Steady-state concentrations of the drug occurred within $\approx 3$ days [7] (3-5 days [29]), with accumulation of $\approx 10 \%$ after intravenous administration $[7,8]$ and $\approx 36 \%$ after oral administration [7]. The absolute bioavailability of delafloxacin after a single oral $450 \mathrm{mg}$ dose was $58.5 \%$. The steadystate volume of distribution of delafloxacin is 30-48 L [7] (40 L [8]; i.e. $\approx$ total body water). Delafloxacin is $\approx 84 \%$ bound to plasma protein, primarily to albumin; plasma protein binding is not significantly affected by renal impairment $[7,8]$. There was no clinically relevant effect of food on the pharmacokinetics of delafloxacin [7, 8, 30].

Delafloxacin is primarily metabolized via glucuronidation (mainly by UGT1A1, UGT1A3 and UGT2B15), with oxidative metabolism representing $\approx 1 \%$ [7] $(<1 \%$ [8]) of an administered dose. Unchanged parent drug is the predominant component in plasma, with no significant circulating metabolites [7, 8]. After a single intravenous dose of radiolabeled delafloxacin, $65 \%$ of the radioactivity is excreted in the urine and $28 \%$ in the faeces [7, 8, 31]. After a radiolabelled oral dose, $50 \%$ is excreted in the urine and $48 \%$ in the faeces [7]. Delafloxacin is excreted in the urine as unchanged drug and glucuronide metabolites; in the faeces, the drug is eliminated as unchanged parent compound [7, 8]. After a single intravenous $300 \mathrm{mg}$ dose, the mean elimination half-life of delafloxacin was $3.7 \mathrm{~h}$ and, after multiple oral doses, ranged from 4.2 to $8.5 \mathrm{~h}$. The mean clearance of delafloxacin was $16.3 \mathrm{~L} / \mathrm{h}$ after a single intravenous $300 \mathrm{mg}$ dose, with renal clearance accounting for 35-45\% of the total clearance [7].

In vitro, delafloxacin has a low potential for drug-drug interactions based on in vitro studies. At clinically relevant concentrations, delafloxacin does not inhibit CYP1A2, CYP2A6, CYP2B6, CYP2C8, CYP2C9, CYP2D6, CYP2E1 and CYP3A4/5 in vitro [7,8], nor does it inhibit UGT1A1 and UGT2B7 [8]. Delafloxacin does not induce CYP1A2, CYP2B6, CYP2C9, CYP2C8, CYP2C19 and CYP3A4/5 in vitro. At clinically relevant concentrations, delafloxacin does not inhibit the transporters MDR1, BCRP, OAT1, OAT3, OCT1, OCT2, OAT1B1, OAT1B3, MATE1, MATE2K and BSEP [7, 8]. The drug is a probable substrate of BCRP [7, 8] and P-gp [7]; it is not a substrate of OAT1, OAT3, OCT1, OCT2, OAT1B1 or OATP [7]. The clinical relevance of coadministration of delafloxacin and P-gp and/ or BCRP inhibitors is unknown [7]. There were no clinically relevant effects on the pharmacokinetics of midazolam (a CYP3A4 substrate) when it was coadministered with delafloxacin after steady state dosing [32]. As with other FQs, the absorption of oral delafloxacin is significantly reduced when it is coadministered with antacids containing aluminium or magnesium, sucralfate, metal irons such as iron, multivitamins containing iron or zinc, formulations containing divalent and trivalent cations or with didanosine buffered tablets for suspension or the paediatric powder for oral solution. Therefore, oral delafloxacin should be taken $\geq 2 \mathrm{~h}$ before or $6 \mathrm{~h}$ after these agents $[7,8]$.

There were no clinically meaningful effects of gender [7, 8], age (adults) [7, 8], race [7], bodyweight [7], body mass index (BMI) [7, 8], disease status [7] or hepatic impairment $[7,8,33]$ on the pharmacokinetics of delafloxacin.

Given its predominantly renal route of elimination, renal impairment has an impact on the clearance of delafloxacin $[34,35]$, including the intravenous vehicle (sulfobutylether$\beta$-cyclodextrin; SBECD) [36]. There is no clinically relevant impact of mild or moderate renal impairment [creatinine clearance $\left(\mathrm{CL}_{\mathrm{CR}}\right) 30-89 \mathrm{~mL} / \mathrm{min}$ ] on the pharmacokinetics of delafloxacin, with no dosage adjustments required in these patients [7, 8, 35]. In patients with severe renal impairment $\left(\mathrm{CL}_{\mathrm{CR}}<30 \mathrm{~mL} / \mathrm{min}\right)$, the dosage of intravenous delafloxacin should be reduced to $200 \mathrm{mg}$ every $12 \mathrm{~h}$ and/or switch to oral delafloxacin $450 \mathrm{mg}$ every $12 \mathrm{~h}$ at the investigators discretion $[7,8]$. In patients with severe renal impairment receiving intravenous delafloxacin, closely monitor serum creatinine levels and estimated glomerular filtration rate (eGFR); if serum creatinine levels increase, consider switching to oral delafloxacin and discontinue delafloxacin if eGFR decreases 
to $<15 \mathrm{~mL} / \mathrm{min} / 1.73 \mathrm{~m}^{2}$ [7]. Accumulation of the intravenous SBECD vehicle occurs in patients with severe renal impairment $[7,8,36]$. Delafloxacin is not recommended in patients with end-stage renal disease (ESRD; due to insufficient information [7]) [7, 8, 34].

\section{Therapeutic Efficacy of Delafloxacin}

The efficacy of fixed-dose intravenous delafloxacin monotherapy (vs intravenous vancomycin + aztreonam) for the treatment of adult patients (aged $\geq 18$ years) with ABSSSIs was evaluated in two large $(n>650 /$ trial $)$, randomized, double-blind, double-dummy, multinational, phase 3 noninferiority trials $[37,38]$. Results from two US, randomized, double-blind, multicentre, phase 2 trials [39, 40] support these phase 3 trials; phase 2 trials are not discussed further.

In phase 3 trials, eligible patients had a diagnosis of ABSSSI defined as cellulitis/erysipelas, wound infection, major cutaneous abscess or burn infection that was characterized by $\geq 75 \mathrm{~cm}^{2}$ of erythema and $\geq 2$ specified signs of systemic infection [37, 38]. Within each trial there were no significant between-group differences in the demographics and clinical characteristics at baseline, including for the distribution of types of ABSSSI, lesion size, systemic and local signs present, and pathogens identified at baseline. In the overall population, $39 \%, 35 \%, 25 \%$ and $<1 \%$ of patients had cellulitis, wound infection, major cutaneous abscess and burn infection, respectively, in study 302 [37]; corresponding rates of these types of ABSSSI in study 303 were $48 \%$, $26 \%, 25 \%$ and $1 \%$ [38]. The overall mean surface area of the infected lesion at baseline was $307 \mathrm{~cm}^{2}$ [37] and $353 \mathrm{~cm}^{2}$ [38], as assessed by digital planimetry. In the overall population of each trial, 32\% [37] and 50\% [38] of patients had a BMI of $\geq 30 \mathrm{~kg} / \mathrm{m}^{2}$; other comorbidities present included hypertension ( 21 and $31 \%$ of patients in studies 302 and 303 ), diabetes (9 and 13\%) and renal impairment (16\% in both trials; of whom, $0.2 \%$ had severe renal impairment or ESRD). In addition, 55\% [37] and 30\% [38] of patients had current or a recent history of drug abuse, including intravenous drug abuse. In terms of baseline cultures, $74.2 \%$ (490/660 enrolled patients) of patients had positive ABSSSI cultures in study 302 , with $S$. aureus $(\approx 66 \%$ of patients in both groups) the most commonly isolated pathogen [37]. Of these isolates, MRSA infections were confirmed in $\approx 34.5 \%$ of cases; $\approx 40 \%$ of $S$. aureus isolates were non-susceptible to levofloxacin, the majority of which were also MRSA. Approximately $17 \%$ of patients had Gram-negative isolates identified [37]. In the other study, amongst the ABSSSI cultures collected from 839 of 850 enrolled patients at baseline, the most commonly identified pathogen was $S$. aureus ( $\approx 57.6 \%$ of isolates), of which $\approx 21 \%$ were MRSA $(96 \%$ susceptible to delafloxacin) and $\approx 26 \%$ were levofloxacin non-susceptible (93\% susceptible to delafloxacin) [38]. Twenty-one percent of patients had Gram-negative isolates identified in baseline cultures [38]. At baseline, $\approx 2.2 \%$ of patients within each trial had bacteraemia [37, 38].

Consistent with current ABSSSI guidance, key exclusion criteria (not all inclusive) included: treatment with a systemic antibacterial in the 14 days prior to enrolment unless specified criteria were met; any underlying chronic conditions at the site of infection that may have complicated the assessment of the response; infections associated with a prosthetic joint or the removal of a prosthetic joint; infections caused by other causes such as human or animal bites, osteomyelitis, diabetic foot ulcer, and necrotizing fasciitis, anaerobic cellulitis or synergistic necrotizing cellulitis; and any infection expected to require other systemic antibacterial agents in addition to study drugs $[37,38]$.

The specific dosage regimens utilized in phase 3 trials are outlined in Table 2, with treatment administered for 5-14 days. The median duration of treatment in the delafloxacin and vancomycin + aztreonam groups was 5 and 5.5 days in study 302 and, in study 303, was 6.5 days in both groups. Where reported, the median duration of treatment with aztreonam or placebo equivalent was 2 days [37].

Delafloxacin (with a switch to oral delafloxacin in study 303) was noninferior to vancomycin + aztreonam for the treatment of ABSSSIs according to FDA- and EMAdefined primary endpoints in the intent-to-treat (ITT) population (primary efficacy population) [Table 2; see table for detailed definitions of these outcomes] [37, 38]. The objective response rate at $48-72 \mathrm{~h}$ did not differ between treatment groups in either trial (FDA specified primary endpoint) and there was no between-group difference for investigatorassessed (IA) clinical cure rate at the follow-up (FU) visit (EMA-defined primary endpoint) [Table 2]. Results in the microbiological ITT (MITT; ITT patients with a baseline pathogen known to cause ABSSSI/eligible pathogen), clinically evaluable (CE; patients who completed activities as per-protocol) and microbiologically evaluable (ME; CE patients with an eligible pathogen) populations were consistent with those in the ITT population, with the noninferiority of delafloxacin to vancomycin + aztreonam demonstrated in all of these populations [37, 38].

Results for other secondary efficacy outcomes were also similar between the delafloxacin and vancomycin + aztreonam treatment groups in each trial in ITT analyses, including IA clinical cure rates and IA success rates (i.e. cure + improvement) at the FU and late FU (LFU) visits (Table 2) [37, 38]. Results in MITT, CE and ME analyses were consistent with these ITT analyses [37, 38]. Additional analyses (as per the EMA statistical analysis plan) also showed no between-group differences for IA clinical cure and success rates at the post-treatment evaluation (PTE) within a window 7-14 days $\left(\mathrm{PTE}_{7-14}\right)$ or 6-15 days 
Table 2 Efficacy of delafloxacin for the treatment of adults with acute bacterial skin and skin structure infections in randomized, double-blind, multinational phase III trials

\begin{tabular}{|c|c|c|c|c|c|c|}
\hline \multirow[t]{2}{*}{ Endpoint } & \multicolumn{3}{|c|}{ Study 302 [37] (\% of pts) } & \multicolumn{3}{|c|}{ Study 303 [38] (\% of pts) } \\
\hline & $\operatorname{DLX}^{\mathrm{a}}(n=331)$ & $\begin{array}{l}\mathrm{VAN}+\mathrm{AZ}^{\mathrm{b}} \\
(n=329)\end{array}$ & $\mathrm{TD}(95 \% \mathrm{CI})$ & $\operatorname{DLX}^{\mathrm{c}}(n=423)$ & $\begin{array}{l}\mathrm{VAN}+\mathrm{AZ}^{\mathrm{d}} \\
(n=427)\end{array}$ & $\mathrm{TD}(95 \% \mathrm{CI})$ \\
\hline OR at $48-72 \mathrm{~h}^{\mathrm{e}}$ & 78.2 & 80.9 & $\begin{array}{l}-2.6(-8.78 \text { to } 3.57) \\
\text { NI }\end{array}$ & 83.7 & 80.6 & $3.1(-2.0$ to 8.3$) \mathrm{NI}$ \\
\hline IA clinical cure at $\mathrm{FU}^{\mathrm{f}}$ & 52.0 & 50.5 & $1.5(-6.11$ to 9.11$) \mathrm{NI}$ & 57.7 & 59.7 & $-2.0(-8.6$ to 4.6$) \mathrm{NI}$ \\
\hline IA success at $\mathrm{FU}^{\mathrm{g}}$ & 81.6 & 83.3 & $\begin{array}{l}-1.7(-7.55 \text { to } 4.13) \\
\text { NI }\end{array}$ & 87.2 & 84.4 & $2.5(-2.2$ to 7.2$)$ \\
\hline $\begin{array}{l}\text { IA clinical cure at } \\
\text { LFU }\end{array}$ & 70.4 & 66.6 & $\begin{array}{l}3.8(-3.27 \text { to } 10.89) \\
\text { NI }\end{array}$ & 67.8 & 71.0 & $-3.1(-9.3$ to 3.1$)$ \\
\hline IA success at LFU & 80.1 & 81.2 & $\begin{array}{l}-1.1(-7.15 \text { to } 4.97) \\
\text { NI }\end{array}$ & 83.5 & 82.2 & $1.3(-3.8$ to 6.3$)$ \\
\hline
\end{tabular}

Results for intent-to-treat analyses (primary efficacy population). All study drugs given IV unless stated otherwise; treatment for 5-14 days

$B L$ baseline, $C F$ clinical failure, $C L_{C R}$ creatinine clearance, $D L X$ delafloxacin, $F U$ follow-up (day $14 \pm 1$ ), $I A$ investigator-assessed, $I V$ intravenous, $L F U$ late FU (day 21-28), NI noninferior to VAN + AZ (based on prespecified criteria), OR objective response, $q 12 \mathrm{~h}$ every $12 \mathrm{~h}, T D$ treatment difference, $V A N+A Z$ vancomycin + aztreonam, $-v e$ negative

${ }^{\mathrm{a}} \mathrm{DLX} 300 \mathrm{mg}$ q12h plus a blinded PL infusion in place of AZ infusion

${ }^{\mathrm{b}}$ VAN $15 \mathrm{mg} / \mathrm{kg} \mathrm{q12} \mathrm{h}$ with a target trough level of $15-20 \mu \mathrm{g} / \mathrm{mL}$ (monitor on day 2 and 6 ) $+\mathrm{AZ} 2 \mathrm{~g}$ q12h until BL cultures proven $-\mathrm{ve}$ for Gram -ve pathogens

${ }^{\mathrm{c}}$ In pts with $\mathrm{CL}_{\mathrm{CR}}>29 \mathrm{~mL} / \mathrm{min}$ : DLX $300 \mathrm{mg} \mathrm{q} 12 \mathrm{~h}$ IV for 6 doses then mandatory switch to oral DLX $450 \mathrm{mg}$ q12h for all remaining doses; in pts with $\mathrm{CL}_{\mathrm{CR}} 15-29 \mathrm{~mL} / \mathrm{min}$ : DLX $200 \mathrm{mg}$ q12h IV for all doses

${ }^{\mathrm{d}}$ Pts with $\mathrm{CL}_{\mathrm{CR}}>29 \mathrm{~mL} / \mathrm{min}$, VAN $15 \mathrm{mg} / \mathrm{kg}$ q12h with a target trough level of $15-20 \mu \mathrm{g} / \mathrm{mL}$ (monitor on day 2 and 6) $+\mathrm{AZ} 2 \mathrm{~g} \mathrm{q} 12 \mathrm{~h}$ until BL cultures proven - ve for Gram - ve pathogens; in pts with $\mathrm{CL}_{\mathrm{CR}} 15-29 \mathrm{~mL} / \mathrm{min}$, renal adjustment of VAN was allowed according to the VAN dosing plan for each site to maintain trough levels $+\mathrm{AZ} 1 \mathrm{~g} \mathrm{q} 12 \mathrm{~h}$ until BL cultures proven - ve for Gram - ve pathogens

${ }^{e}$ FDA defined primary endpoint; defined as $\geq 20 \%$ reduction in the size of the ABSSSI lesion determined by digital planimetry of the leading edge without evidence of CF (based on specified CF criteria, including if planimetry data at this timepoint were not available)

${ }^{\mathrm{f}}$ EMA defined primary endpoint; IA of clinical cure (no remaining/complete resolution of signs or symptoms) at the FU visit

${ }^{\mathrm{g}}$ Success defined as cure or improved and no further antibacterial therapy needed

$\left(\mathrm{PTE}_{6-15}\right)$ of the end of treatment visits [26]. For example, in the pooled ITT efficacy analysis population, respective IA clinical cure and success rates at the $\mathrm{PTE}_{7-14}$ visit in the delafloxacin group $(n=754)$ were $51.2 \%$ (vs $54.0 \%$ in the vancomycin + aztreonam group; $n=756$ ) and $74.8 \%$ (vs $73.7 \%$ ); at the $\mathrm{PTE}_{6-15}$ visit, respective IA cure and success rates in delafloxacin recipients were $55.6 \%$ (vs 56.7\%) and $80.8 \%$ (vs 79.9\%). Results in CE analyses were consistent with these ITT analyses [26].

Overall pathogen eradication rates were also generally similar between the delafloxacin and vancomycin + aztreonam groups ( $n=179$ and 184 [37]; $n=275$ and 277 [38]) in each trial (97.8 vs $98.4 \%$ [37]; 97.8 vs $97.6 \%$ [38]). Amongst those with a baseline MRSA isolate, virtually all patients in the delafloxacin and vancomycin + aztreonam groups had documented or presumed eradication of the pathogen (100 vs $98.5 \%$ [37]; 96.0 vs $97.0 \%$ [38]). All (100\%) [37] and 97\% [38] of delafloxacin recipients who had a levofloxacin non-susceptible $S$. aureus isolate identified at baseline had documented or presumed eradication. There were also similar per pathogen early objective response rates at $48-72 \mathrm{~h}$ and per pathogen microbiological response rates (documented or presumed eradication) at the FU visit in both groups, including for $S$. aureus, MRSA, MSSA, S. anginosus group, K. pneumoniae, E. coli, S. pyogenes, S. lugdunensis and $S$. haemolyticus [37, 38]. These response rates were similar in the delafloxacin and vancomycin + aztreonam groups regardless of whether patients had Gram-positive or Gram-negative infections [37, 38]. Microbiological response rates at the FU visit were also similar in both groups irrespective of whether patients had mono- or polymicrobial Gram-positive or Gram-negative infections, or had mixed Gram-positive and Gram-negative infections [38].

In prespecified subgroup analyses of obese (baseline $\left.\mathrm{BMI} \geq 30 \mathrm{~kg} / \mathrm{m}^{2}\right)$ and non-obese $\left(<30 \mathrm{~kg} / \mathrm{m}^{2}\right)$ patients, there were generally no statistically significant between-group differences in objective response rate at $48-72 \mathrm{~h}$ or IA cure and success rates at the FU or LFU visits in either obese or non-obese patients. However, in obese patients, a statically significant difference in favour of delafloxacin over vancomycin + aztreonam treatment was observed for the IA cure rate at the LFU (71.7 vs $57.4 \%$; between-group difference $14.2 ; 95 \%$ CI $1.34-26.90 ; n=120$ and 94) [37]. 
Pooled analyses in the overall population $(n=1510)$ for primary and secondary outcomes were consistent with those in individual trials for all populations (ITT, MITT, CE, ME) [41]. In prespecified subgroups of patients, there were no significant differences between the delafloxacin and vancomycin + aztreonam for primary and secondary outcomes assessed at 48-72 h, FU or LFU in subgroups of patients with diabetes, a BMI of $\geq 30 \mathrm{~kg} / \mathrm{m}^{2}$ or renal impairment $\left(\mathrm{CL}_{\mathrm{CR}}<90 \mathrm{~mL} / \mathrm{min}\right)$ [41]. There was also no between-group difference in patients with significant drug abuse (abstract) [42] or in those with a history of cardiac or vascular disease (abstract) [43].

In pooled data, there was no effect on the efficacy of delafloxacin based on the presence of baseline risk factors related to the host, pathogen and disease, including aged $>65$ years, BMI $\geq 30 \mathrm{~kg} / \mathrm{m}^{2}$, diabetes, prior antibacterial treatment, MRSA infection, bacteraemia, polymicrobial infection, $\geq 3$ signs of systemic infection, type of infection (wound infection and erythema $\geq 4$ th percentile) [abstract] [44]. Treatment success rates in higher-risk ( $\geq 3$ risk factors; $n=625)$ and lower-risk ( $<3$ risk factors; $n=881)$ groups were high in both groups at the end-of-treatment visit ( 90.3 vs $90.8 \%)$ and FU visit (82.9 vs $86.0 \%)$.

\section{Tolerability of Delafloxacin}

Intravenous and/or oral delafloxacin was generally well tolerated in clinical trials in patients with ABSSSIs discussed in Sect. 4. Discussion focuses on pooled safety data from the pivotal phase 3 trials $(n=741$ and 751 in the delafloxacin and vancomycin + aztreonam groups) [45, 46], including a pooled analysis of adverse events of special interest (AESI) [46]. Baseline characteristics in the overall population were: $11 \%$ had diabetes, $16 \%$ had renal impairment, $\approx 42 \%$ had a BMI of $\geq 30 \mathrm{mg} / \mathrm{kg}^{2}$ and $\approx 29 \%$ had history of hepatitis B or C [45]. The mean exposure to delafloxacin was 6.8 days (range $0.5-14.0$ days; vs mean of 6.6 days in vancomycin + aztreonam group), with $39.5 \%$ of patients receiving $\leq 10$ doses (vs 39.0\%) [45].

In the pooled analysis, $45.1 \%$ and $47.7 \%$ of patients in the delafloxacin and vancomycin + aztreonam groups experienced a treatment-emergent adverse event (TEAE), most of which were of mild to moderate severity $(<4 \%$ of patients experienced a severe TEAE) and few patients (1.8 vs $3.5 \%$ ) discontinued treatment because of these adverse events. Of the TEAEs, $22.1 \%$ in the delafloxacin group and $26.1 \%$ in the vancomycin + aztreonam group were considered to be treatment related (TRAEs), with $0.8 \%$ and $2.4 \%$ of patients discontinuing treatment because of these events. The most common (i.e. frequency $\geq 2 \%$ in either group) TRAEs occurring in delafloxacin and vancomycin + aztreonam recipients were nausea ( 6.1 vs $4.3 \%$ ), diarrhoea (6.1 vs $2.0 \%$ ) and skin and subcutaneous tissue disorders ( 0.9 vs $4.7 \%$; pruritus, urticaria, dermatitis, rash). Relatively few patients in the delafloxacin and vancomycin + aztreonam groups experienced a serious TRAE ( 0.3 vs $0.5 \%)$. The tolerability profiles with delafloxacin and vancomycin + aztreonam treatment were consistent, irrespective of baseline patient characteristics (age, gender, race/ethnicity, degree of renal impairment, diabetes status, BMI or history of infectious hepatitis B or C) [45].

\subsection{Adverse Events of Special Interest Associated with Fluoroquinolones}

AESI associated with the FQ class occurred in $7.0 \%$ and $9.2 \%$ of delafloxacin and vancomycin + aztreonam recipients, with most of these events of mild to moderate severity and no apparent increase in the risk of such events between the delafloxacin and vancomycin groups [46]. Of note, comparison of delafloxacin with a non-FQ comparator group (i.e. AESI were not associated FQ use) permits comparison of AESI rates with delafloxacin to that in the general population, especially in patients with a high burden of underlying comorbidities such as the population enrolled in pivotal trials discussed in Sect. 4.

In the pooled phase 3 trial analysis, the rates of AESI were relatively low (typically $<1 \%$ ) in both treatment groups, with most of these events of mild to moderate severity [46]. In the delafloxacin and vancomycin + aztreonam groups, very few patients experienced treatment-related hepatic-related events ( 2.2 vs $2.7 \%)$, potential myopathy $(0.9$ vs $2.7 \%)$, hyperglycaemia ( 0.3 vs $0.1 \%$ ), hypoglycaemia ( 0.1 vs $0.3 \%$ ), potential peripheral neuropathy ( 0.1 vs $0.3 \%)$, potential QT prolongation (0 vs $0.1 \%$ ), potential tendon disorder (no cases in either group), Clostridium difficile-associated diarrhoea $(0.1$ vs $0 \%)$, convulsions ( 0 vs $0.1 \%$ ) or potential phototoxicity ( 0 vs $0 \%$ ). Overall, the rate of hepatic TRAEs (hypertransaminasaemia, increased transaminases or liver function test abnormalities) [2.2 vs $2.7 \%$, was similar in the delafloxacin and vancomycin + aztreonam groups, with no patients meeting Hy's Law criteria. No patients receiving ongoing treatment for seizures or with an untreated history of seizures were included in the pivotal phase 3 trials [46].

FQ use has been associated with disabling and potentially irreversible serious AEs, including tendonitis and tendon rupture (bilateral in some instances), peripheral neuropathy (e.g. paraesthesia, hypoesthesia, dysesthesia and weakness), CNS adverse reactions (e.g. anxiety, agitation, convulsions, depression, hallucinations, increased intracranial pressure, insomnia, nervousness and tremors) and exacerbation of myasthenia gravis $[7,8]$. Discontinue delafloxacin treatment immediately and avoid the use of FQs in patients who experience any of these serious adverse reactions $[7,8]$. The use of delafloxacin should be avoided in patients with a 
known history of tendon disorders [7] (contraindicated [8]) or who have experienced tendinitis or tendon rupture [7], a known history of myasthenia gravis [7] (not recommended [8]) or a history of peripheral neuropathy [7]. As with all FQs, in patients with known or suspected CNS disorders or in the presence of other risk factors that may predispose to seizures or lower the seizure threshold, delafloxacin should only be used when the benefits exceed the risks $[7,8]$. FQs have been associated with disturbances in blood glucose, including hypoglycaemia and hyperglycaemia, usually in patients with diabetes receiving concomitant treatment with oral antihyperglycaemic agents (e.g. sulfonylureas) or with insulin. Severe cases of hypoglycaemia resulting in coma or death have been reported (with other FQs [7]). In diabetic patients, careful monitoring of blood glucose levels is recommended. There are no data available on severe cases of hypoglycaemia resulting in coma or death after delafloxacin use $[7,8]$. If a hypoglycaemic reaction occurs during delafloxacin treatment, discontinue delafloxacin therapy and initiate appropriate treatment immediately [7].

\section{Dosage and Administration of Delafloxacin}

Delafloxacin is approved in several countries for the treatment of ABSSSI; specific indications may vary between countries. In the EU, delafloxacin is indicated for the treatment of ABSSSI in adults when it is considered inappropriate to use other antibacterial agents that are commonly recommended for the initial treatment of these infections [8]. In the USA, delafloxacin is indicated for the treatment of adults with ABSSSI or community-acquired bacterial pneumonia caused by designated susceptible bacteria [7]. The recommended dosage of delafloxacin is $300 \mathrm{mg}$ every $12 \mathrm{~h}$ administered as a $60-\mathrm{min}$ intravenous infusion. A switch to oral delafloxacin $450 \mathrm{mg}$ every $12 \mathrm{~h}$ is possible at the discretion of the physician. The total treatment duration is 5-14 days $[7,8]$. Consult local prescribing information for detailed information, including specific indications, contraindications, precautions and warnings, potential drug interactions and use in special patient populations.

\section{Place of Delafloxacin in the Management of ABSSSIs}

Current 2014 Infectious Diseases Society of America [47] and 2018 World Society of Emergency Surgery/Surgical Infection Society Europe [1] guidelines for managing severe ABSSSIs recommend several first-line empirical treatment options, including ceftaroline, clindamycin, linezolid, telavancin and vancomycin (considered the first-line parenteral treatment for serious MRSA infections in hospitalized patients $[3,47])$. The approval of delafloxacin is too recent for the drug to have been included in these guidelines. Other newer antibacterials that have recently been approved for the treatment of ABSSSIs include dalbavancin, tedizolid, oritavancin and daptomycin. Notably, it is critical that, as is the case with all antibacterials, newer antibacterials such as delafloxacin should be used in a judicious manner to minimize the risk of the emergence of drug resistance, as has occurred with older antibacterials. Antibacterial therapy for ABSSSIs is primarily empirical until a pathogen(s) is identified. The choice of antibacterial is dependent on several factors, including the site of infection (specific pathogens are typically associated with certain wound sites; e.g. MRSA and MSSA are the predominant pathogens involved in wound infections following cardiothoracic surgery), drug characteristics (e.g. drug-drug interactions, ability to transition from intravenous to oral therapy, efficacy and safety profile), patient characteristics (e.g. presence of comorbidities, age), causative pathogen(s) and local antimicrobial resistance patterns [48-50].

Delafloxacin is a novel anionic FQ, with its unique chemical structure resulting in enhanced activity in acidic environments characteristic of skin abscesses and dual equipotent inhibition of topoisomerase IV and DNA gyrase (Sect. 2). Delafloxacin exhibits excellent in vitro activity against a broad spectrum of clinically relevant Gram-positive, including MRSA strains ( \pm levofloxacin-resistant strains and those with mutations in the QRDR), and Gram-negative bacteria (Sect. 2.1), and is associated with a low risk for the emergence of resistance (Sects. 2.1, 2.2). Older FQs (e.g. ciprofloxacin, levofloxacin and moxifloxacin) are not commonly used for treating ABSSSIs caused by MRSA, primarily reflecting the requirement for additional antibacterials to cover skin infections caused by FQ-resistant organisms and also because of their safety profile, especially in patients with comorbidities and the elderly [3-5, 10]. Delafloxacin treatment was associated with high rates of microbiological cure against MRSA isolates ( \pm levofloxacin-resistant strains and those with mutations in the QRDR) in pivotal phase 3 trials (Sect. 2.1); this suggests that delafloxacin is a good option for treating ABSSSIs caused by $S$. aureus isolates, including MRSA strains in which high rates of ciprofloxacin and levofloxacin nonsusceptibility are observed [17].

Several other drug characteristics are also important in determining the choice of empirical therapy such as the potential for drug-drug interactions and availability of intravenous and oral formulations for ease of transition from the in-patient to the out-patient setting. Delafloxacin is available as an intravenous and oral formulation (Sect. 6), does not alter the QTc interval to a clinically relevant extent (Sect. 2.4) and has a favourable pharmacokinetic profile (good oral bioavailability, low potential for drug-drug 
interactions and a convenient twice-daily regimen) [Sect. 3]. Dosage adjustment is required in patients with severe renal impairment with intravenous, but not oral, delafloxacin (Sect. 3).

In primary ITT analyses in the pivotal global phase 3 trials in adults with ABSSSIs, intravenous delafloxacin (with switch to oral delafloxacin after six doses in one study) was noninferior to intravenous vancomycin + aztreonam in terms of the primary FDA- and EMA-defined endpoints (Sect. 4). Results in other study populations (MITT, CE and ME populations), prespecified subgroups (based on age, gender, race/ethnicity, degree of renal impairment, diabetes status, BMI or history of infectious hepatitis B or C) and in pooled analyses were consistent with those in the ITT population. There was no between-group difference in overall pathogen eradication rates ( $\approx 89 \%$ in both groups in both trials), with similar per pathogen early objective response rates at $48-72 \mathrm{~h}$ and per pathogen microbiological response rates at the FU visit in both groups, including for $S$. aureus, MRSA, MSSA, S. anginosus group, K. pneumoniae, E. coli, S. pyogenes, $S$. lugdunensis and S. haemolyticus isolates. Amongst the limitations of these trials were the low number of burn infections and surgical wounds, and the number of Gramnegative pathogens was limited by the use of the current ABSSSI definition that favours Gram-positive infections.

Delafloxacin was generally well tolerated in clinical trials in adults with ABSSSIs, with relatively few patients discontinuing treatment because of TRAEs and most TRAEs of mild to moderate severity (Sect. 5). The tolerability profile of delafloxacin was similar regardless of baseline patient characteristic, including age, gender, race/ethnicity, degree of renal impairment, diabetes status, BMI or history of infectious hepatitis $\mathrm{B}$ or $\mathrm{C}$. The most common TRAEs (incidence $>1 \%$ ) occurring during delafloxacin treatment were nausea $(6.1 \%)$ and diarrhoea (6.1\%). Moreover, the rates of AESI in delafloxacin recipients were generally low $(\leq 2 \%)$, including rates of treatment-related hepatic AEs, potential myopathy, dysglycaemic episodes, potential peripheral neuropathy, potential QT prolongation, potential tendon disorder and potential phototoxicity (Sect. 5.1). There was no apparent increase in the risk of AESI between the delafloxacin and vancomycin groups. To date, unlike some other FQs, delafloxacin has not been associated with cases of QT prolongation or phototoxicity and, given its low brain penetration, delafloxacin potentially may be associated with less neurotoxicity than other FQs [2].

In conclusion, ongoing clinical experience with delafloxacin treatment in patients with ABSSSIs will help to define its relative role with respect to other antibacterials in this clinical setting. In pivotal phase 3 trials in adult patients with ABSSSIs, including in those with comorbid diseases, intravenous delafloxacin monotherapy ( \pm oral switch) was noninferior to intravenous vancomycin + aztreonam in terms of clinical efficacy and was generally well tolerated. Given its unique chemical structure that confers novel properties relative to other FQ and its broad spectrum of activity against common clinically relevant Gram-positive pathogens, including against MRSA strains $( \pm$ FQ-resistance mutations), and Gram-negative pathogens, intravenous delafloxacin ( \pm oral switch) provides a novel emerging option for the treatment of adult patients with ABSSSIs.

\section{Data Selection Delafloxacin: 534 records identified \\ Duplicates removed \\ Excluded during initial screening (e.g. press releases; news reports; not relevant drug/indication; preclinical study; reviews; case reports; not randomized trial) \\ Excluded during writing (e.g. reviews; duplicate data; small patient number; nonrandomized/phase I/II trials) \\ Cited efficacy/tolerability articles \\ Cited articles not efficacy/tolerability \\ Search Strategy: EMBASE, MEDLINE and PubMed from 1946 to present. Clinical trial registries/databases and websites were also searched for relevant data. Key words were delafloxa- cin, Quifenix, Baxdela, ABSSSI, acute bacterial skin and skin structure infections. Records were limited to those in English language. Searches last updated 29 June 2020}

Acknowledgments The preparation of this review was not supported by any external funding. During the peer review process, Menarini Group was offered an opportunity to comment on this article. Changes resulting from such comments were made on the basis of scientific and editorial merit.

Funding The preparation of this review was not supported by any external funding.

\section{Compliance with ethical standard}

Conflict of interest Lesley Scott is a salaried employee of Adis International Ltd/Springer Nature, is responsible for the article content and declares no relevant conflicts of interest.

Open Access This article is licensed under a Creative Commons Attribution-NonCommercial 4.0 International License, which permits any non-commercial use, sharing, adaptation, distribution and reproduction in any medium or format, as long as you give appropriate credit to the original author(s) and the source, provide a link to the Creative Commons licence, and indicate if changes were made. The images or other third party material in this article are included in the article's Creative Commons licence, unless indicated otherwise in a credit line to the material. If material is not included in the article's Creative Commons licence and your intended use is not permitted by statutory regulation or exceeds the permitted use, you will need to obtain permission directly from the copyright holder. To view a copy of this licence, visit http://creativecommons.org/licenses/by-nc/4.0/. 


\section{References}

1. Sartelli M, Guirao X, Hardcastle TC, et al. WSES/SIS-E consensus conference: recommendations for the management of skin and soft-tissue infections. Worl J Emerg Surg. 2018;13:58.

2. Bassetti M, Peghin M, Castaldo N, et al. The safety of treatment options for acute bacterial skin and skin structure infections. Expert Opin Drug Saf. 2019;18(8):635-50.

3. Golan Y. Current treatment options for acute skin and skin-structure infections. Clin Infect Dis. 2019;68(Suppl 3):S206-S212212.

4. Mogle BT, Steele JM, Thomas SJ, et al. Clinical review of delafloxacin: a novel anionic fluoroquinolone. J Antimicrob Chemother. 2018;73(6):1439-51.

5. Kocsis B, Domokos J, Szabo D. Chemical structure and pharmacokinetics of novel quinolone agents represented by avarofloxacin, delafloxacin, finafloxacin, zabofloxacin and nemonoxacin. Ann Clin Microbiol Antimicrob. 2016;15(1):34.

6. Van Bambeke F. Delafloxacin, a non-zwitterionic fluoroquinolone in phase III of clinical development: evaluation of its pharmacology, pharmacokinetics, pharmacodynamics and clinical efficacy. Future Microbiol. 2015;10(7):1111-23.

7. Melinta Therapeutics. Baxdela (delafloxacin) tablets, for oral use; Baxdela (delafloxacin) for injection, for intravenous use: US prescribing information. 2019. https://baxdela.com/docs/baxde la-prescribing-information.pdf. Accessed 16 Mar 2020.

8. European Medicines Agency. Quofenix (delafloxacin): summary of product characteristics. 2019. https://www.ema.europa.eu/. Accessed 16 Mar 2020.

9. Bassetti M, Pecori D, Cojutti P, et al. Clinical and pharmacokinetic drug evaluation of delafloxacin for the treatment of acute bacterial skin and skin structure infections. Expert Opin Drug Metab Toxicol. 2017;13(11):1193-200.

10. Jorgensen SCJ, Mercuro NJ, Davis SL, et al. Delafloxacin: place in therapy and review of microbiologic, clinical and pharmacologic properties. Infect Dis Ther. 2018;7(2):197-21717.

11. Emami S, Shafiee A, Foroumadi A. Quinolones: recent structural and clinical developments. Iran J Pharm Res. 2005;3:123-36.

12. Tulkens PM, Van Bambeke F, Zinner SH. Profile of a novel anionic fluoroquinolone-delafloxacin. Clin Infect Dis. 2019;68(Suppl 3):S213-S22.

13. Hayashi N, Nakata Y, Yazaki A. New findings on the structurephototoxicity relationship and photostability of fluoroquinolones with various substituents at position 1. Antimicrob Agents Chemother. 2004;48(3):799-803.

14. Nilius AM, Shen LL, Hensey-Rudloff D, et al. In vitro antibacterial potency and spectrum of ABT-492, a new fluoroquinolone. Antimicrob Agents Chemother. 2003;47(10):3260-9.

15. Pfaller MA, Sader HS, Rhomberg PR, et al. In vitro activity of delafloxacin against contemporary bacterial pathogens from the United States and Europe, 2014. Antimicrob Agents Chemother. 2017;61(4):e02609-e2616.

16. Flamm RK, Rhomberg PR, Huband MD, et al. In vitro activity of delafloxacin tested against isolates of Streptococcus pneumoniae, Haemophilus influenzae, and Moraxella catarrhalis. Antimicrob Agents Chemother. 2016;60(10):6381-5.

17. McCurdy S, Lawrence L, Quintas M, et al. In vitro activity of delafloxacin and microbiological response against fluoroquinolone-susceptible and nonsusceptible Staphylococcus aureus isolates from two phase 3 studies of acute bacterial skin and skin structure infections. Antimicrob Agents Chemother. 2017;61(9):e00772-e817.

18. Rossolini G, Zinzi D, Nuti A, et al. Delafloxacin: activity against fastidious organisms tested by EUCAST vs CLSI methodology [abstract plus poster]. In: ASM Microbe Meeting. 2018.
19. Siala W, Mingeot-Leclercq MP, Tulkens PM, et al. Comparison of the antibiotic activities of daptomycin, vancomycin, and the investigational fluoroquinolone delafloxacin against biofilms from Staphylococcus aureus clinical isolates. Antimicrob Agents Chemother. 2014;58(11):6385-97.

20. Bauer J, Siala W, Tulkens PM, et al. A combined pharmacodynamic quantitative and qualitative model reveals the potent activity of daptomycin and delafloxacin against Staphylococcus aureus biofilms. Antimicrob Agents Chemother. 2013;57(6):2726-37.

21. Remy JM, Marra A, Duffy E. Bactericidal activity of delafloxacin against recent isolates of Staphylococcus aureus [poster no. 272]. In: ASM Microbe Meeting. 2016.

22. Almer LS, Hoffrage JB, Keller EL. In vitro and bactericidal activities of ABT-492, a novel fluoroquinolone, against gram-positive and gram-negative organisms. Antimicrob Agents Chemother. 2004;48(7):2711-77.

23. Remy JM, Tow-Keogh CA, McConnell TS, et al. Activity of delafloxacin against methicillin-resistant Staphylococcus aureus: resistance selection and characterization. J Antimicrob Chemother. 2012;67(12):2814-20.

24. Lepak AJ, Andes DR. In vivo pharmacodynamic target assessment of delafloxacin against Staphylococcus aureus, Streptococcus pneumoniae, and Klebsiella pneumoniae in a murine lung infection model. Antimicrob Agents Chemother. 2016;60(8):4764-9.

25. Zhao M, Lepak AJ, Marchillo $\mathrm{K}$, et al. In vivo pharmacodynamic target determination for delafloxacin against Klebsiella pneumoniae and Pseudomonas aeruginosa in the neutropenic murine pneumonia model. Antimicrob Agents Chemother. 2019;63(10):e01131-e1219.

26. European Medicines Agency. Quofenix (delafloxacin): public assessment report. 2019. https://www.ema.europa.eu/. Accessed 20 May 2020.

27. Litwin JS, Benedict MS, Thorn MD, et al. A thorough QT study to evaluate the effects of therapeutic and supratherapeutic doses of Delafloxacin on cardiac repolarization. Antimicrob Agents Chemother. 2015;59(6):3469-73.

28. Dawe RS, Ferguson J, Ibbotson S, et al. Lack of phototoxicity potential with delafloxacin in healthy male and female subjects: comparison to lomefloxacin. Photochem Photobiol Sci. 2018;17(6):773-80

29. Hoover R, Hunt T, Benedict M, et al. Safety, tolerability, and pharmacokinetic properties of intravenous delafloxacin after single and multiple doses in healthy volunteers. Clin Ther. 2016;38(1):53-655.

30. Hoover R, Hunt T, Benedict M, et al. Single and multiple ascending-dose studies of oral delafloxacin: effects of food, sex, and age. Clin Ther. 2016;38(1):39-52.

31. McEwen A, Lawrence L, Hoover R, et al. Disposition, metabolism and mass balance of delafloxacin in healthy human volunteers following intravenous administration. Xenobiotica. 2015;45(12):1054-62.

32. Paulson SK, Wood-Horrall RN, Hoover R, et al. The pharmacokinetics of the CYP3A substrate midazolam after steady-state dosing of delafloxacin. Clin Ther. 2017;39(6):1182-90.

33. Hoover R, Marbury TC, Preston RA, et al. Clinical pharmacology of delafloxacin in patients with hepatic impairment. J Clin Pharmacol. 2017;57(3):328-35.

34. Hoover R, Alcorn H Jr, Lawrence L, et al. Pharmacokinetics of intravenous delafloxacin in patients with end-stage renal disease. J Clin Pharmacol. 2018;58(7):913-9.

35. Hoover RK, Alcorn H Jr, Lawrence L, et al. Delafloxacin pharmacokinetics in subjects with varying degrees of renal function. J Clin Pharmacol. 2018;58(4):514-21.

36. Hoover RK, Alcorn H Jr, Lawrence L, et al. Clinical pharmacokinetics of sulfobutylether- $\beta$-cyclodextrin in patients 
with varying degrees of renal impairment. J Clin Pharmacol. 2018;58(6):814-22.

37. Pullman J, Gardovskis J, Farley B, et al. Efficacy and safety of delafloxacin compared with vancomycin plus aztreonam for acute bacterial skin and skin structure infections: a phase 3, double-blind, randomized study. J Antimicrob Chemother. 2017;72(12):3471-80.

38. O'Riordan W, McManus A, Teras J, et al. A comparison of the efficacy and safety of intravenous followed by oral delafloxacin with vancomycin plus aztreonam for the treatment of acute bacterial skin and skin structure infections: a phase 3, multinational, double-blind, randomized study. Clin Infect Dis. 2018;67(5):657-66.

39. O'Riordan W, Mehra P, Manos P, et al. A randomized phase 2 study comparing two doses of delafloxacin with tigecycline in adults with complicated skin and skin-structure infections. Int $\mathbf{J}$ Infect Dis. 2015;30:67-73.

40. Kingsley J, Mehra P, Lawrence LE, et al. A randomized, doubleblind, phase 2 study to evaluate subjective and objective outcomes in patients with acute bacterial skin and skin structure infections treated with delafloxacin, linezolid or vancomycin. J Antimicrob Chemother. 2016;71(3):821-9.

41. Giordano PA, Pogue JM, Cammarata S. Analysis of pooled phase III efficacy data for delafloxacin in acute bacterial skin and skin structure infections. Clin Infect Dis. 2019;68(Suppl 3):S223-S232.

42. Overcash JS, O'Riordan W, Quintas M, et al. Treatment of acute bacterial skin and skin structure infections (ABSSSI) in patients with significant drug abuse: outcomes from global phase 3 studies of delafloxacin (DLX) [abstract no. 470]. Open Forum Infect Dis. 2019;6(Suppl 2):S230.
43. Oguchi G, Beasley R, Lawrence L, et al. Outcomes in patients with history of cardiac or vascular disease $(\mathrm{CV})$ during treatment of acute bacterial skin and skin structure infection (ABSSSI) with delafloxacin (DLX) vs. vancomycin/aztreonam (VAN/ AZ) [abstract no. 2377]. Open Forum Infect Dis. 2018;5(Suppl 1):S708.

44. Piza B, Nizzardo A, Nuti A, et al. Risk factors (derived from the host, pathogen and disease) did not affect the efficacy of delafloxacin (DLX) monotherapy in acute bacterial skin and skin structure infections (ABSSSI) trials (abstract). In: ECCMID. 2020.

45. Bassetti M, Hooper D, Tillotson G. Analysis of pooled phase 3 safety data for delafloxacin in acute bacterial skin and skin structure infections. Clin Infect Dis. 2019;68(Suppl 3):S233-S240.

46. Lodise T, Corey R, Hooper D, et al. Safety of delafloxacin: focus on adverse events of special interest. Open Forum Infect Dis. 2018;5(10):ofy220.

47. Stevens DL, Bisno AL, Chambers HF, et al. Practice guidelines for the diagnosis and management of skin and soft tissue infections: 2014 update of the Infectious Diseases Society of America. Clin Infect Dis. 2014;59(2):147-59.

48. Jaffa RK, Pillinger KE, Roshdy D, et al. Novel developments in the treatment of acute bacterial skin and skin structure infections. Expert Opin Pharmacother. 2019;20(12):1493-502.

49. Pulido-Cejudo A, Guzmán-Gutierrez M, Jalif-Montaño A, et al. Management of acute bacterial skin and skin structure infections with a focus on patients at high risk of treatment failure. Ther Adv Infect Dis. 2017;4(5):143-61.

50. Bassetti M, Magnasco L, Del Puent F, et al. Role of new antibiotics in the treatment of acute bacterial skin and skin structure infections. Curr Opin Infect Dis. 2020;33(2):110-20. 